Pacific Journal of Mathematics

SLOSHING FREQUENCIES IN A HALF-SPACE BY KELVIN 


\title{
SLOSHING FREQUENCIES IN A HALF-SPACE BY KELVIN INVERSION
}

\author{
B. Andreas Troesch
}

The frequencies of the free oscillations of an incompressible and inviscid fluid in a half-space with circular and strip-like aperture are determined by first mapping the halfspace into a bounded domain by the Kelvin inversion. Upper bounds for the eigenfrequencies are then obtained by a Rayleigh-Ritz procedure. The results compare quite well with previous results, which were obtained by less elementary tools.

1. Introduction. The free oscillations of an incompressible, inviscid liquid in a container have been investigated extensively (see [1] and [6], and the references given there). In this paper we determine approximations to the sloshing frequencies for fluid in a covered half-space, where the free surface is a circular aperture. The problem represents the limiting case of free sloshing motions in arbitrary axially symmetric containers with the same free surface, and its solution furnishes universal upper bounds for the sloshing frequencies in these containers [11]. Recently, quite accurate results have been obtained in [4], including rigorous error bounds for the frequencies. Although the underlying geometry and the problem statement are quite simple, the solution with the error bounds turned out to be rather intricate.

We will present here an alternate method and show that quite acceptable upper bounds may be computed with considerably more modest tools. There are two reasons that account for the simplifications. First of all we will choose coordinate functions in the Rayleigh-Ritz procedure, which do not satisfy the natural boundary conditions of the variational formulation of the problem. This leads to considerable simplifications, but it precludes, on the other hand, the ready computation of lower bounds. Secondly, the complications arising from the fact that the domain is unbounded will be avoided by using a Kelvin inversion. The problem is solved for the image domain of a large spherical bowl, and only in the final result is the bowl radius assumed to tend to infinity.

The corresponding variational formulation can still be established without difficulty, although the problem is slightly more complicated after the Kelvin transformation (cf. eqs. (2.5) to (2.8) below). In this setting, the problem is solved by a Rayleigh-Ritz method. The matrix elements turn out to be rational numbers (with the 
exception of a few logarithmic terms) and can therefore be computed easily and accurately.

The mathematical problem, both in its original form and after the Kelvin transformation, is stated in $\S 2$. The next section describes in detail how the original differential equation and the boundary conditions transform under the Kelvin inversion. The $\S 4$ establishes the variational principle, and $\S 5$ deals with the choice of the coordinate functions in the Rayleigh-Ritz procedure. In $\S 6$ it is indicated how the integrals, which represent the elements in the matrix eigenvalue problem, are evaluated.

The discussion of the limiting process from the large spherical bowl to a half-space is taken up in $\S 7$. The next section comments on the two-dimensional sloshing problems in a half-space with a strip-like aperture, and the numerical results for both the axially symmetric and the plane case are given in the last section.

2. Problem statement. The sloshing motion of a fluid in a half-space with a circular free surface of radius $a$ is described by the following eigenvalue problem for the velocity potential $f(x, y, z)[4]$, where the eigenvalue appears only in the boundary condition.

The Laplace equation is satisfied in the lower half-space $z<0$

$$
\nabla^{2} f=0,
$$

and the boundary conditions on the horizontal $x$ - $y$-plane are

$$
\begin{array}{ll}
\frac{\partial f}{\partial z}(x, y, 0)=\lambda f(x, y, 0) & \text { for } \quad 0 \leqq x^{2}+y^{2}<a^{2} \\
\frac{\partial f}{\partial z}(x, y, 0)=0 & \text { for } \quad a^{2}<x^{2}+y^{2}<\infty .
\end{array}
$$

The eigenvalues $\lambda$ are related to the frequencies of oscillation $\omega$ and to the acceleration of gravity $g$ by

$$
\lambda=\omega^{2} / g \text {. }
$$

In the approach taken in this paper, the eqs. (2.1) to (2.3) are satisfied inside a large spherical bowl and on the part of the $x-y$ plane which cuts the bowl, whereas on the spherical part of the bowl the usual boundary condition for a finite container is imposed (cf. Fig. 1), namely that the normal derivative of the velocity potential vanishes

$$
\frac{\partial f}{\partial n}=0 \quad \text { on } \quad S_{1}
$$

If the Kelvin inversion is applied, then the problem takes on the 
following form, in which it is actually solved. Determine the set of eigenvalues $\lambda$ for the eigenvalue problem for the function $g$ :

$$
\nabla^{2} g=0
$$

in the indented sphere $C_{3}$ of radius $\alpha / 2$ (see Fig. 1); the boundary condition

$$
g+a \frac{\partial g}{\partial r}=0
$$

is satisfied on the upper half-sphere, except on the indentation of radius $\rho$. On the indentation

$$
\rho \frac{\partial g}{\partial n}=g
$$

holds ( $n$ is the outer normal to the shaded domain). Furthermore,

$$
(1-\cos \theta)\left(g+a \frac{\partial g}{\partial r}\right)=2 \lambda a g
$$

holds on the lower half of the sphere $C_{3}$.

The derivation of this form of the problem is carried out in the next section.

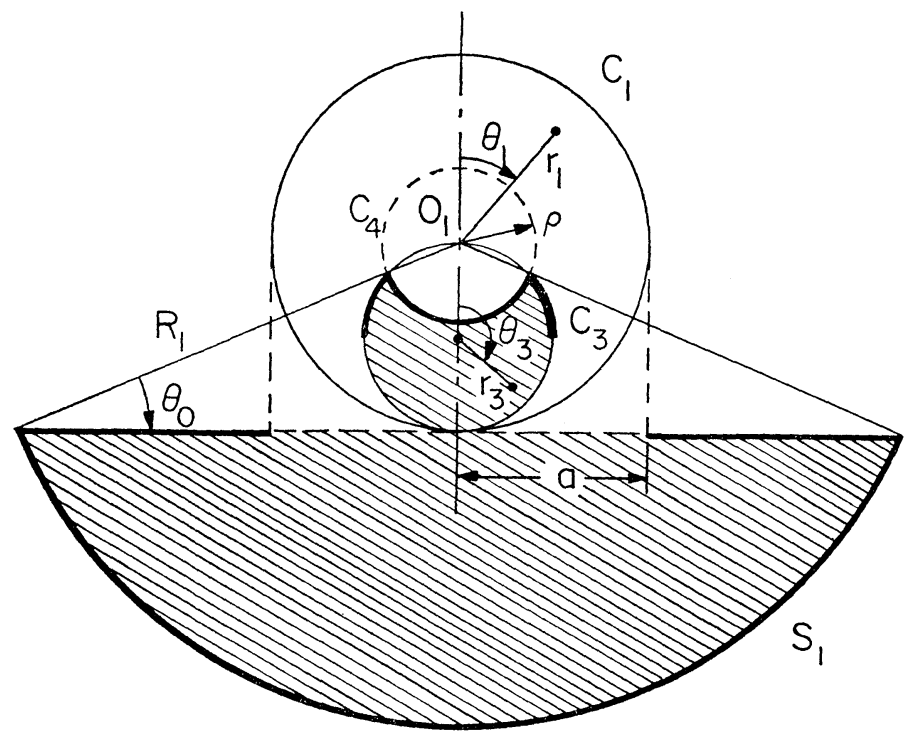

FIG. 1 The geometry of the Kelvin inversion.

A remark should be added about the singularity which occurs at the transition from the free surface to the covered part of the 
spherical bowl, i.e., at $x^{2}+y^{2}=a^{2}, z=0$. From the physical point of view we have to stipulate that the singularity be weaker than a source or a sink (cf. [10], p. 59 and p. 80). This is also the appropriate mathematical condition for the problem: as P. Henrici has shown in a striking example (cf. [4], p. 296), the spectrum of the problem changes its character drastically (there appear finite eigenvalues $\lambda$ of infinite multiplicity), if the singularity is too strong. Since the singularity at the rim is a local phenomenon, the solution of the dock problem (waves in a half-space covered by a rigid halfplane) is applicable. This solution is given in [3] (and also used in [8], p.323) in terms of the complex velocity potential: there is a logarithmic singularity at the rim. For the Rayleigh-Ritz procedure used below the exact nature of the sufficiently weak singularity need not be taken into account. The coordinate functions (see §5) will probably represent the true eigenfunctions rather poorly near the rim; but it is known that the Rayleigh-Ritz method nevertheless furnishes good upper bounds for the eigenvalues. Under some circumstances it is advisable to isolate the singular behavior of a solution by including in the set of coordinate functions a function with the proper singularity. However, the computation of the matrix elements in $\S 6$ would then become considerably more involved.

Incidentally, the solution of the dock problem ([3], [8]) has been used in [12] to obtain the asymptotic distribution of the eigenvalues for the problem considered here.

3. The Kelvin inversion on the sphere $C_{1}$. The original problem is first restated in spherical coordinates $r_{1}, \theta_{1}, \varphi_{1}$ with the origin at the center $O_{1}$ of the sphere of inversion $C_{1}$. The $x-y$-plane is then described by

$$
r_{1} \cos \theta_{1}+a=0,
$$

and its normal unit vector in the $z$-direction by

$$
\vec{n}=\left(\cos \theta_{1},-\sin \theta_{1}, 0\right) \text {. }
$$

Hence the boundary conditions, eqs. (2.2), (2.3), and (2.4), for the harmonic function

$$
f_{1}\left(r_{1}, \theta_{1}, \varphi_{1}\right)=f(x, y, z)
$$

become, on the plane (3.1),

$$
\frac{\partial f_{1}}{\partial r_{1}} \cos \theta_{1}-\frac{\sin \theta_{1}}{r_{1}} \frac{\partial f_{1}}{\partial \theta_{1}}=\lambda f_{1} \quad \text { for } \frac{3 \pi}{4}<\theta_{1} \leqq \pi
$$




$$
\frac{\partial f_{1}}{\partial r_{1}} \cos \theta_{1}-\frac{\sin \theta_{1}}{r_{1}} \frac{\partial f_{1}}{\partial \theta_{1}}=0 \quad \text { for } \frac{\pi}{2}+\theta_{0} \leqq \theta_{1}<\frac{3 \pi}{4},
$$

and furthermore,

$$
\frac{\partial f_{1}}{\partial r_{1}}=0
$$

on the large spherical bowl $S_{1}$ of radius $R_{1}$. For the meaning of the angle $\theta_{0}$ see Fig. 1 , where it appears greatly exaggerated, of course.

Next we map the lower half-space into the sphere $C_{3}$ by reciprocal radii, i.e., by the transformation

$$
r_{2}=\frac{a^{2}}{r_{1}}, \quad \theta_{2}=\theta_{1}, \quad \varphi_{2}=\varphi_{1} .
$$

For the equation of the sphere $C_{3}$ we then obtain

$$
a \cos \theta_{2}+r_{2}=0 \quad \text { for } \frac{\pi}{2} \leqq \theta_{2} \leqq \pi
$$

and for the spherical indentation $C_{4}$ with radius $\rho$

$$
r_{2}=\rho
$$

where $\rho=a^{2} / R_{1}$.

If we transform $f_{1}$ in the usual manner by introducing ([9] p. 140)

$$
f_{2}\left(r_{2}, \theta_{2}, \varphi_{2}\right)=\frac{a^{2}}{r_{2}} f_{1}\left(\frac{a^{2}}{r_{2}}, \theta_{2}, \varphi_{2}\right),
$$

then $f_{2}$ is again a harmonic function

$$
\nabla^{2} f_{2}=0 \text {. }
$$

The boundary conditions on $C_{3}$ turn out to be

$$
\begin{aligned}
r_{2} \cos \theta_{2} f_{2}+r_{2}^{2} \cos \theta_{2} \frac{\partial f_{2}}{\partial r_{2}}+r_{2} \sin \theta_{2} \frac{\partial f_{2}}{\partial \theta_{2}}= & -\lambda \alpha^{2} f_{2} \\
& \text { for } \frac{3 \pi}{4}<\theta_{2} \leqq \pi,
\end{aligned}
$$

$$
\begin{aligned}
\cos \theta_{2} f_{2}+r_{2} \cos \theta_{2} \frac{\partial f_{2}}{\partial r_{2}}+\sin \theta_{2} \frac{\partial f_{2}}{\partial \theta_{2}}= & 0 \\
& \text { for } \frac{\pi}{2}+\theta_{0} \leqq \theta_{2}<\frac{3 \pi}{4},
\end{aligned}
$$

and finally on the indentation

$$
r_{2} \frac{\partial f_{2}}{\partial r_{2}}+f_{2}=0 \quad \text { for } \quad r_{2}=\rho .
$$


Clearly, the proper coordinates to solve (3.2) to (3.4) are the spherical coordinates with the origin at the center of the sphere $C_{3}$ of radius $a / 2$, i.e.,

$$
\begin{aligned}
r_{3} \cos \theta_{3} & =r_{2} \cos \theta_{2}+\frac{a}{2} \\
r_{3} \sin \theta_{3} & =r_{2} \sin \theta_{2} \\
\varphi_{3} & =\varphi_{2} .
\end{aligned}
$$

On the surface $C_{3}$ the relations

$$
\begin{aligned}
& \frac{\partial r_{3}}{\partial r_{2}}=\sin \left(\frac{\theta_{3}}{2}\right) \\
& \frac{\partial r_{3}}{\partial \theta_{2}}=-a \sin \left(\frac{\theta_{3}}{2}\right) \cos \left(\frac{\theta_{3}}{2}\right) \\
& \frac{\partial \theta_{3}}{\partial r_{2}}=\frac{2}{a} \cos \left(\frac{\theta_{3}}{2}\right) \\
& \frac{\partial \theta_{3}}{\partial \theta_{2}}=2 \sin ^{2}\left(\frac{\theta_{3}}{2}\right)
\end{aligned}
$$

hold. Hence, the boundary conditions (3.3), (3.4) transform, after some straightforward simplifications, to

$$
\left(1-\cos \theta_{3}\right)\left(f_{3}+a \frac{\partial f_{3}}{\partial r_{3}}\right)=2 \lambda a f_{3}
$$

on the lower half-sphere, denoted by $S_{l}$ :

$$
r_{3}=\frac{a}{2}, \frac{\pi}{2}<\theta_{3} \leqq \pi,
$$

and to

$$
f_{3}+a \frac{\partial f_{3}}{\partial r_{3}}=0
$$

on the upper half-sphere:

$$
r_{3}=\frac{a}{2}, \quad \bar{\theta} \leqq \theta_{3}<\frac{\pi}{2},
$$

where $f_{3}\left(r_{3}, \theta_{3}, \varphi_{3}\right)=f_{2}\left(r_{2}, \theta_{2}, \varphi_{2}\right)$ satisfies

$$
\nabla^{2} f_{3}=0
$$

inside the indented sphere $C_{3}$. The angle $\bar{\theta}>0$ excludes the indentation from $C_{3}$. The boundary condition (3.5) on the indentation is best left in the old coordinate system. Thus the problem statement 
of $\S 2$ is established, and the subscripts can be dropped for the spherical coordinates.

4. The variational principle. It is not difficult to find by inspection the variational principle which leads to the eigenvalue problem (2.5) to (2.8).

Let the functional $L[g]$ be defined as

$$
L[g]=N[g] / D[g]
$$

with

$$
\begin{gathered}
N[g]=\iiint_{V}|\nabla g|^{2} d V+\frac{1}{a} \iint_{S} g^{2} d S-\frac{1}{\rho} \iint_{C_{4}} g^{2} d S, \\
D[g]=\iint_{S_{l}} \frac{2}{(1-\cos \theta)} g^{2} d S .
\end{gathered}
$$

In $N[g]$ the volume integral is taken over the indented sphere, one surface integral is taken over the surface $S$ of the sphere $C_{3}$, without the indentation, and the other surface integral over the indentation $C_{4}$. In $D[g]$ the surface integral extends over the lower halfsphere. We show now by the standard method of the calculus of variations that the stationary values of $L$ are the eigenvalues $\lambda$ of the problem $(2.5)$ to $(2.8)$ :

$$
\begin{array}{r}
\iiint_{V} \nabla g \cdot \nabla \delta g d V+\frac{1}{a} \iint_{S} g \delta g d S-\frac{1}{\rho} \iint_{C_{4}} g \delta g d S \\
-\lambda \iint_{S_{l}} \frac{2}{(1-\cos \theta)} g \delta g d S=0,
\end{array}
$$

and using Green's theorem

$$
\begin{aligned}
-\iiint_{V} \delta g V^{2} g d V & +\iint_{S} \delta g \frac{\partial g}{\partial n} d S+\iint_{C_{4}} \delta g \frac{\partial g}{\partial n} d S+\frac{1}{a} \iint_{S} \delta g g d S \\
& -\frac{1}{\rho} \iint_{C_{4}} \delta g g d S-\lambda \iint_{S_{l}} \frac{2}{(1-\cos \theta)} \delta g g d S=0 .
\end{aligned}
$$

The differential equation and the boundary conditions (2.5) to (2.8) follow now at once. We notice, in particular, that the boundary conditions turn out to be natural boundary conditions. This enables us to choose as admissible function in the variational principle any continuous function with piecewise continuous first derivatives for which the integrals exist.

5. The Rayleigh-Ritz method. We now proceed to find the stationary values of (4.1) by the Rayleigh-Ritz method. Since the true 
eigenfunctions of the problem are harmonic, it is desirable to choose as coordinate functions a complete system of harmonic functions (cf. [6] p. 240, [7] p. 96). An obvious choice for $g$ is then

$$
\begin{aligned}
g=\sum_{n=m}^{n=N} \alpha_{n} f_{n} \cos m \varphi=\sum_{n=m}^{n=N} \alpha_{n}\left(\frac{2 r}{a}\right)^{n} P_{n}^{m}(\cos \theta) \cos m \varphi & \\
& m=1,2, \cdots .
\end{aligned}
$$

The functions $P_{n}^{m}$ are the spherical harmonics, and the $\alpha_{n}$ 's are the free coefficients in the Rayleigh-Ritz method.

For the sloshing modes without radial nodal lines, the above expression for $g$ is not the best possible choice, because for $m=0$ the original problem possesses the eigenvalue $\lambda=0$ with a constant eigenfunction. Under the inversion, this function does not remain constant and should be included in the set of coordinate functions. Although the Rayleigh-Ritz method would still furnish upper bounds for the eigenvalues, the results would be considerably less accurate without this function, especially for small $N$. For this additional function the index $n=-1$ is chosen, in order not to disturb the standard notation for the Legendre polynomials $P_{n}$. By the inversion on the sphere one obtains

$$
f_{-1}=\frac{a}{r_{2}}=\left(\frac{r^{2}}{a^{2}}+\frac{1}{4}-\frac{r}{a} \cos \theta\right)^{-1 / 2}
$$

If $N \rightarrow \infty$, this coordinate function would of course be redundant. For $m=0$ we now choose

$$
g=\alpha_{-1} f_{-1}+\sum_{n=0}^{n=N} \alpha_{n}\left(\frac{2 r}{a}\right)^{n} P_{n}(\cos \theta)
$$

Since the trigonometric functions are orthogonal, the problem is decomposed into its symmetry classes $m=0,1,2, \cdots$, and each symmetry class can be investigated separately. This fact has already been taken into account in the coordinate functions introduced above. The factor $\cos m \varphi$ could of course be replaced by $\sin m \varphi$ without altering the subsequent steps, and it then follows that all eigenvalues for $m \geqq 1$ are double eigenvalues.

We are now ready to find the upper bounds for the functional $L[g]$ in eq. (4.1). In order to take advantage of the fact that all the coordinate functions are harmonic, the eq. (4.2) is transformed by Green's theorem

$$
N[g]=\iint_{S} g\left(\frac{\partial g}{\partial r}+\frac{g}{a}\right) d S+\iint_{C_{4}} g\left(\frac{\partial g}{\partial n}-\frac{g}{\rho}\right) d S .
$$

For each symmetry class $m$, eq. (5.1) leads to 


$$
\begin{aligned}
L_{m}[g]= & N_{m}[g] / D_{m}[g] \\
N_{m}[g]= & \sum_{j, k} \alpha_{j} \alpha_{k}\left\{\iint_{S} f_{j}\left(\frac{\partial f_{k}}{\partial r}+\frac{f_{k}}{a}\right) \cos ^{2} m \varphi d S\right. \\
& \left.+\iint_{C_{4}} f_{j}\left(\frac{\partial f_{k}}{\partial n}-\frac{f_{k}}{\rho}\right) \cos ^{2} m \varphi d S\right\} \\
D_{m}[g]= & \sum_{j, k} \alpha_{j} \alpha_{k} \iint_{S_{l}} f_{j} f_{k} \frac{2}{(1-\cos \theta)} \cos ^{2} m \varphi d S .
\end{aligned}
$$

If we minimize $L$ with respect to the coefficients $\alpha$ ([7] p.96), we obtain the algebraic eigenvalue problem of the form

$$
\operatorname{det}(A-\lambda B)=0 \text {. }
$$

6. The computation of the matrix elements. The matrix elements are, after suppressing the common factors from the integration over $\varphi$

$$
\begin{aligned}
a_{j k}= & \int_{\bar{\theta}}^{\pi} f_{j}\left(\frac{\partial f_{k}}{\partial r}+\frac{f_{k}}{a}\right) \frac{a^{2}}{4} \sin \theta d \theta \\
& +\int_{\pi / 2+\theta_{0}}^{\pi} f_{j}\left(\frac{\partial f_{k}}{\partial n}-\frac{f_{k}}{\rho}\right) \rho^{2} \sin \theta_{2} d \theta_{2} \\
b_{j k}= & \int_{\pi / 2}^{\pi} f_{j} f_{k} \frac{2}{(1-\cos \theta)} \frac{a^{2}}{4} \sin \theta d \theta .
\end{aligned}
$$

It is convenient to leave the second integral of $a_{j k}$ in the spherical coordinates centered at $O_{1}$. In spite of their appearance, both matrices $A$ and $B$ are symmetrical.

The matrix elements $a_{j k}$ and $b_{j k}$ are evaluated under the assumption that $\theta_{0}=0$, and hence $\bar{\theta}_{0}=0, \rho=0$. These assumptions will be justified in the next section. For all $m$, and $j \geqq m, k \geqq m$

$$
f_{k}=\left(\frac{2 r}{a}\right)^{k} P_{k}^{m}(\cos \theta),
$$

and setting $\cos \theta=x$, the eqs. (6.1) and (6.2) simply become

$$
a_{j k}=\frac{a}{4} \int_{-1}^{1}(2 k+1) P_{j}^{m}(x) P_{k}^{m}(x) d x=\frac{a}{2} \frac{(j+m) !}{(j-m) !} \delta_{j k}
$$

with the Kronecker symbol $\delta_{j k}($ see [5], p. 116), and

$$
b_{j k}=\frac{a^{2}}{4} \int_{-1}^{0} P_{j}^{m}(x) P_{k}^{m}(x) \frac{2}{(1-x)} d x .
$$

For $m=0$, there are the additional elements $a_{-1, k}, a_{-1-1}, b_{-1, k}, b_{-1,-1}$. Using eq. (5.2) for $r=a / 2$, we obtain 


$$
\begin{aligned}
& b_{-1, k}=\frac{a^{2}}{4} \int_{-1}^{0} \frac{2 \sqrt{2}}{(1-x)^{3 / 2}} P_{k}(x) d x, \quad k=0,1, \cdots \\
& b_{-1,-1}=\frac{a^{2}}{4} \int_{-1}^{0} \frac{4}{(1-x)^{2}} d x=\frac{a^{2}}{2} .
\end{aligned}
$$

Both integrals in the elements $a_{k,-1}$ and $a_{-1,-1}$ vanish, since we find from eq. (5.2) that on $C_{3}$

$$
\frac{\partial f_{-1}}{\partial r}+\frac{f_{-1}}{a}=0
$$

and on the indentation $C_{4}$

$$
\frac{\partial f_{-1}}{\partial n}-\frac{f_{-1}}{\rho}=0
$$

As the matrix $A$ is symmetrical, the elements $a_{-1, k}$ also vanish; indeed, in this case, the two integrals are equal in magnitude and of opposite sign.

It remains to evaluate the integrals in eqs. (6.4) and (6.5). If the Legendre functions $P$ are expressed in terms of $(1-x)$, as given in [5] p. 111, and the division is carried out, then the finite series can be integrated termwise. The results look cumbersome, if they are spelled out, but they are easily implemented recursively in a computer program.

7. The limit of an infinitely large spherical bowl. The integrals in eq. (6.1) have been evaluated for $\theta_{0}=0$, and hence $\bar{\theta}=0$, $\rho=0$. This is permissible, because the contribution of the indentation in the sphere $C_{3}$ to the matrix elements can be made arbitrarily small. All the coordinate functions, with the exception of $f_{-1}$, are regular in the neighborhood of the point $O_{1}$. By shrinking the radius $\rho$ of the indentation $C_{4}$ to zero, we can make the contribution of the indentation to the matrix elements $\alpha_{j k}$ as small as we wish. In the elements $a_{k,-1}$ and $a_{-1,-1}$ the integrand vanishes even for finite $\rho$ according to (6.7) and (6.8). This result is of course expected, as $\lambda=0$ should remain an eigenvalue for the problem after the Kelvin inversion.

The roots $\lambda$ of the characteristic polynomial are all nonnegative and depend continuously on its coefficients, i.e., on the elements of the matrices $A$ and $B$. Therefore, if the indentation is ignored entirely, the Rayleigh-Ritz eigenvalues differ arbitrarily little from those for a small indentation, or, in terms of the original problem, from the Rayleigh-Ritz eigenvalues of the lower half-space bounded by a sphere of large radius $R_{1}$. 
8. The planar case. For the case where the sloshing motion takes place in two dimensions, the same method can be applied. The free surface is now a strip of width $2 a$ perpendicular to the plane in which the velocity vectors lie. Fig. 1 still depicts the situation, except that the inversion takes place on a circle rather than on a sphere. The sloshing frequencies now represent universal upper bounds for sloshing in a canal where the fluid motion is perpendicular to the canal axis.

The mathematical problem which describes the motion is, in terms of the velocity potential $f$, as follows:

$$
\begin{aligned}
\nabla^{2} f & =0 & \text { for } z<0 \\
\frac{\partial f}{\partial z}(x, 0) & =\lambda f(x, 0) & \text { for } 0<|x|<a \\
\frac{\partial f}{\partial z}(x, 0) & =0 & \text { for } a<|x|,
\end{aligned}
$$

and, as we replace the half-space by a large cylinder $S_{1}$,

$$
\frac{\partial f}{\partial n}=0
$$

After the Kelvin inversion, we obtain

$$
\nabla^{2} g=0
$$

in the indented circle $C_{3}$, and as boundary conditions

$$
\frac{\partial g}{\partial n}=0
$$

on the upper half-circle of $C_{3}$ and on the indentation $C_{4}$,

$$
(1-\cos \theta) \frac{\partial g}{\partial n}=2 \lambda g
$$

on the lower half- circle of $C_{3}$, denoted again by $S_{l}$.

Eqs. (8.5) to (8.7) are the Euler-Lagrange equation and the natural boundary conditions of the variational problem, which consists of making the functional

$$
L[g]=\iint|\nabla g|^{2} d A / \int_{S_{l}} \frac{2}{(1-\cos \theta)} g^{2} d S
$$

stationary. The double integral is extended over the indented circle (see Fig. 1).

The obvious choice for the coordinate functions for the Rayleigh- 
Ritz method is now

$$
f_{n}=\left(\frac{2 r}{a}\right)^{n} \sin n \theta, \quad n=1,2, \cdots
$$

for the odd modes,

$$
f_{n}=\left(\frac{2 r}{a}\right)^{n} \cos n \theta, \quad n=0,1,2, \cdots
$$

for the even modes.

There is no need to introduce a special function corresponding to eq. (5.2), since for the inversion by reciprocal radii in two dimensions a constant function transforms into a constant, and this term is included by setting $n=0$ for the even modes. The limit $R_{1} \rightarrow \infty$ also causes no problem.

A simple calculation furnishes for the matrix elements in

$$
\operatorname{det}(A-\lambda B)=0
$$

the results

$$
a_{j k}=j \pi \delta_{j k}
$$

and

$$
b_{j k}=(-1)^{j+k} a\left(C_{|j-k|} \pm C_{j+k}\right)
$$

with the upper sign for the even, the lower sign for the odd modes. The integrals

$$
C_{n}=\int_{0}^{\pi / 2} \frac{\cos n \varphi}{1+\cos \varphi} d \varphi=\int_{0}^{\pi / 4} \frac{\cos 2 n \varphi}{\cos ^{2} \varphi} d \varphi
$$

which appear in $b_{j k}$ are readily evaluated as finite sums.

9. Numerical results. The numerical results for the upper bounds of the sloshing eigenvalues in a half-space are summarized in Table 1. They are based on the solution of a 16 by 16 matrix eigenvalue problem. Since the matrix $A$ is diagonal in both the plane and the axi-symmetric case (cf. eqs. (6.3) and (8.8)), the eigenvalue problem is of the simplest type.

By observing the trend of the results as the order of the matrices $A$ and $B$ is increased to 16 by 16 , and by applying an Aitken extrapolation, the eigenvalues can be improved, namely by a fraction of a percent for $\lambda_{1}$ and by a few percent for $\lambda_{5}$. However, the extrapolated values are no longer guaranteed to be upper bounds.

In Table 2 the present results are compared with previous results for three representative examples. 
TABLE 1

\begin{tabular}{|c|c|c|c|c|c|c|c|c|c|}
\hline & \multicolumn{2}{|c|}{ Planar case } & \multicolumn{7}{|c|}{ axi-symmetric case } \\
\hline & $\begin{array}{c}\text { odd } \\
\text { modes }\end{array}$ & $\begin{array}{l}\text { even } \\
\text { modes }\end{array}$ & $m=0$ & $m=1$ & $m=2$ & $m=3$ & $m=4$ & $m=5$ & $m=6$ \\
\hline$a \lambda_{1}$ & 2.009 & 3.462 & 4.133 & 2.759 & 4.130 & 5.415 & 6.651 & 7.857 & 9.041 \\
\hline$a \lambda_{2}$ & 5.148 & 6.665 & 7.385 & 5.915 & 7.376 & 8.764 & 10.103 & 11.409 & 12.688 \\
\hline$a \lambda_{3}$ & 8.332 & 9.885 & 10.630 & 9.097 & 10.602 & 12.047 & 13.45 & 14.82 & 16.16 \\
\hline$a \lambda_{4}$ & 11.583 & 13.17 & 13.94 & 12.32 & 13.86 & 15.35 & 16.80 & 18.22 & 19.62 \\
\hline$a \lambda_{5}$ & 14.98 & 16.62 & 17.40 & 15.64 & 17.22 & 18.76 & 20.26 & 21.73 & 23.18 \\
\hline
\end{tabular}

Upper bounds for the sloshing eigenvalues in a half-space, with strip-like aperture of width $2 a$ in the planar case, and with circular aperture of radius $a$ in the axi-symmetric case.

TABLE 2

\begin{tabular}{l|c|c|c}
\hline & Present results & Ref. [2] & Ref. [4] \\
\hline Planar case & & & \\
odd mode, $a \lambda_{1}$ & 2.009 & 2.018 & 2.006 \\
\hline $\begin{array}{l}\text { axi-symmetric case } \\
m=1, a \lambda_{3}\end{array}$ & 9.097 & 9.25 & 9.033 \\
$m=5, a \lambda_{5}$ & 21.73 & - & 21.14 \\
\hline
\end{tabular}

Comparison of three typical examples with the upper bounds from [2] and [4].

Added in proof. The reader should also consult the paper by J. W. Miles, On the eigenvalue problem for fluid sloshing in a halfspace, Z. Angew. Math. Phys., 23 (1972), 861-869.

\section{REFERENCES}

1. H. N. Abramson, The Dynamic Behavior of Liquids in Moving Containers, NASA SP-106, National Aeronautics and Space Administration, Washington, 1966.

2. B. Budiansky, Sloshing of liquids in circular canals and spherical tanks, J. Aero/Space Sci., 27 (1960), 161-173.

3. K. O. Friedrichs and H. Lewy, The dock problem, Comm. Pure Appl. Math., 1 (1948), 135-148.

4. P. Henrici, B. A. Troesch, and L. Wuytack, Sloshing frequencies for a half-space with circular or strip-like aperture, Z. Angew. Math. Phys., 21, (1970), 285-318.

5. E. Jahnke and F. Emde, Tables of Functions, Fourth Edition, Dover Publications, New York, 1945.

6. N. N. Moiseev, Introduction to the theory of oscillations of liquid-containing bodies, Advances in Applied Mechanics, 8 (1964), 233-289.

7. N. N. Moiseev and A. A. Petrov, The calculation of free oscillations of a liquid in a motionless container, Advances in Applied Mechanics, 9 (1966), 91-154.

8. H. Rubin, The dock of finite extent, Comm. Pure Appl. Math., 7 (1954), 317-344. 
9. A. Sommerfeld, Partial Differential Equations in Physics, Academic Press, New York and London, 1964.

10. J. J. Stoker, Water Waves, Interscience Publishers, Inc., New York, 1957.

11. B. A. Troesch, An isoperimetric sloshing problem, Comm. Pure Appl. Math., 18 (1965), 319-338.

12. B. A. Troesch and H. R. Troesch, A remark on the sloshing frequencies in a halfspace, Z. Angew. Math. Phys., 23 (1972), 703-711.

Received May 25, 1972. This research was supported, in part, by the National Science Foundation under Grant GP-22587.

UNIVERSITY OF SOUTHERN CALIFORNIA 


\section{PACIFIC JOURNAL OF MATHEMATICS}

\section{EDITORS}

D. Gilbarg AND J. MILGRAM

Stanford University

Stanford, California 94305

\section{R. A. Beaumont}

University of Washington

Seattle, Washington 98105

\section{J. DUGUNDJI*}

Department of Mathematics University of Southern California Los Angeles, California 90007

RICHARD ARENS

University of California Los Angeles, California 90024

\section{ASSOCIATE EDITORS}
E. F. BECKENBACH
B. H. NEUMANN
F. WOLF
K. YoshidA

\section{SUPPORTING INSTITUTIONS}

\author{
UNIVERSITY OF BRITISH COLUMBIA \\ CALIFORNIA INSTITUTE OF TECHNOLOGY \\ UNIVERSITY OF CALIFORNIA \\ MONTANA STATE UNIVERSITY \\ UNIVERSITY OF NEVADA \\ NEW MEXICO STATE UNIVERSITY \\ OREGON STATE UNIVERSITY \\ UNIVERSITY OF OREGON \\ OSAKA UNIVERSITY
}

\author{
UNIVERSITY OF SOUTHERN CALIFORNIA \\ STANFORD UNIVERSITY \\ UNIVERSITY OF TOKYO \\ UNIVERSITY OF UTAH \\ WASHINGTON STATE UNIVERSITY \\ UNIVERSITY OF WASHINGTON \\ AMERICAN MATHEMATICAL SOCIETY \\ NAVAL WEAPONS CENTER
}

The Supporting Institutions listed above contribute to the cost of publication of this Journal, but they are not owners or publishers and have no responsibility for its content or policies.

Mathematical papers intended for publication in the Pacific Journal of Mathematics should be in typed form or offset-reproduced, (not dittoed), double spaced with large margins. Underline Greek letters in red, German in green, and script in blue. The first paragraph or two must be capable of being used separately as a synopsis of the entire paper. Items of the bibliography should not be cited there unless absolutely necessary, in which case they must be identified by author and Journal, rather than by item number. Manuscripts, in duplicate if possible, may be sent to any one of the four editors. Please classify according to the scheme of Math. Rev. Index to Vol. 39. All other communications to the editors should be addressed to the managing editor, Richard Arens, University of California, Los Angeles, California, 90024.

50 reprints are provided free for each article; additional copies may be obtained at cost in multiples of 50 .

The Pacific Journal of Mathematics is issued monthly as of January 1966. Regular subscription rate: $\$ 48.00$ a year (6 Vols., 12 issues). Special rate: $\$ 24.00$ a year to individual members of supporting institutions.

Subscriptions, orders for back numbers, and changes of address should be sent to Pacific Journal of Mathematics, 103 Highland Boulevard, Berkeley, California, 94708.

PUBLISHED BY PACIFIC JOURNAL OF MATHEMATICS, A NON-PROFIT CORPORATION

Printed at Kokusai Bunken Insatsusha (International Academic Printing Co., Ltd.), 270, 3-chome Totsuka-cho, Shinjuku-ku, Tokyo 160, Japan.

* C. DePrima will replace J. Dugundji until August 1974.

Copyright (C) 1973 by

Pacific Journal of Mathematics

All Rights Reserved 


\section{Pacific Journal of Mathematics}

\section{Vol. 47, No. $2 \quad$ February, 1973}

David Parham Bellamy, Composants of Hausdorff indecomposable continua; a mapping approach ........................ 303

Colin Bennett, A Hausdorff-Young theorem for rearrangement-invariant spaces ...........................................

Roger Daniel Bleier and Paul F. Conrad, The lattice of closed ideals and $a^{*}$-extensions of an abelian l-group ...

Ronald Elroy Bruck, Jr., Nonexpansive projections on subsets of Banach

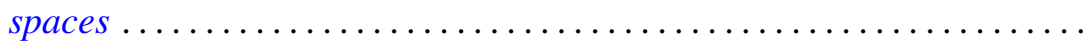

Robert C. Busby, Centralizers of twisted group algebras ............. 357

M. J. Canfell, Dimension theory in zero-set spaces ................ 393

John Dauns, One sided prime ideals ........................ 401

Charles F. Dunkl, Structure hypergroups for measure algebras . . . . . . . . . 413

Ronald Francis Gariepy, Geometric properties of Sobolev mappings ...... 427

Ralph Allen Gellar and Lavon Barry Page, A new look at some familiar spaces of intertwining operators ...........................

Dennis Michael Girard, The behavior of the norm of an automorphism of the

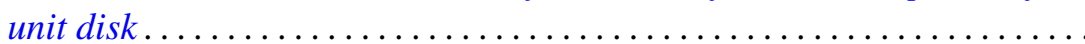

George Rudolph Gordh, Jr., Terminal subcontinua of hereditarily

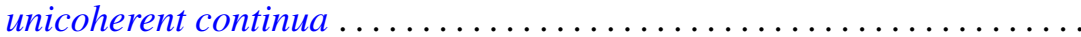

Joe Alston Guthrie, Mapping spaces and cs-networks. .

Neil Hindman, The product of $F$-spaces with $P$-spaces . 473

M. A. Labbé and John Wolfe, Isomorphic classes of the spaces $C_{\sigma}(S)$

Ernest A. Michael, On k-spaces, $k_{R}$-spaces and $k(X) \ldots$

Donald Steven Passman, Primitive group rings .

C. P. L. Rhodes, A note on primary decompositions of a pseudovaluation ...

Muril Lynn Robertson, A class of generalized functional differential equations

Ruth Silverman, Decomposition of plane convex sets. $I$.

Ernest Lester Stitzinger, On saturated formations of solvable Lie algebras................................

B. Andreas Troesch, Sloshing frequencies in a half-space by Kelvin inversion ...

L. E. Ward, Fixed point sets .

Michael John Westwater, Hilbert transforms, and a problem in scattering

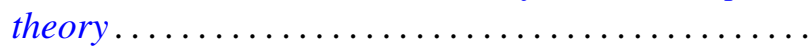

Misha Zafran, On the spectra of multipliers... 